\title{
Intraoperative inspection alone is a reliable guide to the choice of surgical procedure for enteroenteric fistulas in Crohn's disease
}

\author{
Zhen $\mathrm{Guo}^{1}$, Xingchen $\mathrm{Cai}^{2}$, Ruiqing $\mathrm{Liu}^{1}$, Jianfeng Gong ${ }^{1}$, Yi $\mathrm{Li}^{1}$, Lei Cao ${ }^{1}$, Weiming Zhu ${ }^{1}$ \\ ${ }^{1}$ Department of General Surgery, Jinling Hospital, Medical School of Nanjing University, Nanjing, ${ }^{2}$ Department of General Surgery, Jinling \\ Hospital, Nanjing Medical University, Nanjing, China
}

Background/Aims: Resection of the diseased segment and suture of the victim segment is recommended for enteroenteric fistula in Crohn's disease (CD). The main difficulty in this procedure remains reliable diagnosis of the victim segment, especially for fistulas found intraoperatively and inaccessible on endoscopic examination. We aimed to explore whether intraoperative inspection alone is reliable. Methods: Patients undergoing conservative surgery between 2011 and 2016 for enteroenteric fistulas complicating CD were identified from a prospectively maintained database. Patients were divided according to whether the victim segment was evaluated by preoperative endoscopy + intraoperative inspection (PI group) or by intraoperative inspection alone (I group). Outcomes were compared. Results: Of 65 patients eligible for the study, 37 were in in the PI group and 28 were in the I group. The baseline characteristics were similar between the groups, except for the rate of emergency surgery (0/37 in PI group vs. 5/28 in I group, $P=0.012)$. Fistulas involved more small intestines (4/37 in PI group vs. 15/28 in I group, $P<0.001)$ and fewer sigmoid colons ( $17 / 37$ in PI group vs. $4 / 28$ in I group, $P=0.008)$ in I group due to accessibility with endoscopy. No difference was found in postoperative complications, stoma rates, postoperative recurrence, or disease at the repair site between the 2 groups $(P>0.05)$. Conclusions: For fistulas found intraoperatively and inaccessible on endoscopic examination, intraoperative inspection was a reliable guide when choosing between en bloc resection and a conservative procedure. (Intest Res 2018;16:282-287)

Key Words: Crohn disease; Enteroenteric fistulas; Surgical procedure

\section{INTRODUCTION}

Enteroenteric fistulas occur in $5 \%$ to $30 \%$ of patients with $\mathrm{CD}$, and often require surgical treatment. ${ }^{1,2}$ Usually, enteroenteric fistulas in $\mathrm{CD}$ arise from a diseased segment of bowel and extend to a victim segment that is free from CD. As repeated resection is always required for recurrent disease in $\mathrm{CD}$, in order to preserve as much intestine as possible, en bloc resection of fistulas (resection of both segments) is

Received September 25, 2017. Revised November 21, 2017.

Accepted December 4, 2017. Published online February 22, 2018

Correspondence to Weiming Zhu, Department of General Surgery, Jinling

Hospital, Medical School of Nanjing University, No. 305 East Zhongshan

Road, Nanjing 210002, China. Tel: +86-25-80860036, Fax: +86-25-

80860220, E-mail: juwiming@nju.edu.cn not recommended. Instead, a more conservative procedure (resection of diseased segment and suture of the victim segment) is advocated. ${ }^{3}$

Previous studies confirmed the conservative procedure was safe when suture repair was performed on the CD-free victim segment. ${ }^{4-8}$ However, when the sutured segment was affected by $\mathrm{CD}$, the risk of postoperative fistula or disease recurrence was increased. ${ }^{8}$ Therefore, the evaluation of the victim segment is essential for the decision on whether suture repair can be performed. Preoperative endoscopy with biopsies is the most accurate method for assessment of the victim segment, but it is only possible when the fistula is accessible, as in an ileosigmoid or coloduodenal location. However, more than $20 \%$ of enteroenteric fistulas are incidental

๑ Copyright 2018. Korean Association for the Study of Intestinal Diseases. All rights reserved.

This is an Open Access article distributed under the terms of the Creative Commons Attribution Non-Commercial License (http://creativecommons.org/licenses/by-nc/4.0)

which permits unrestricted non-commercial use, distribution, and reproduction in any medium, provided the original work is properly cited. 
intraoperative findings. ${ }^{7}$ For fistulas that are difficult to reach with endoscopy, intraoperative inspection (macroscopic observation and tactile assessment) remains the most feasible approach to the choice of surgical procedure. The present study aimed to determine whether intraoperative inspection alone is a reliable guide compared with preoperative endoscopy + intraoperative inspection when choosing between en bloc resection and a conservative procedure in CD complicated by an enteroenteric fistula.

\section{METHODS}

\section{Patients}

Approval from the Institutional Review Board of Jinling Hospital was obtained (2015NZKY-25), and the study was performed according to the principles of the Declaration of Helsinki. Eligible patients gave written informed consent. All patients undergoing surgical treatment at the IBD center in Jinling Hospital for enteroenteric fistulas complicating CD between January 2011 and August 2016 were identified from a prospectively maintained database. When additional data were required, medical records were reviewed. Only patients receiving suture closure of the defect on the victim segment were included in this retrospective study. The diagnosis of CD was endoscopically and histologically confirmed, and the diagnosis of enteroenteric fistula was confirmed by radiographic, endoscopic, and/or intraoperative findings. The following information was collected: sex, age at surgery, disease duration, disease phenotype according to the Montreal classification, smoking history, family history, preoperative medical treatment, and initial clinical manifestation. Details of surgical procedures, diameter of defects, postoperative complications, and CD recurrence were also obtained.

Preoperative total abdominal CT was routinely performed on all patients in our center, and preoperative colonoscopy was performed on those undergoing elective intestinal resection. Gastroduodenoscopy was performed when coloduodenal fistula was suspected. Fistula, abscess or phlegmon, and a star sign on CT, fistula and pseudopolyp on endoscopy, and fistula on fluoroscopic contrast studies were considered positive findings. When fistula was diagnosed or suspected, the intestine around the fistula was evaluated using endoscopy with biopsies if possible. Patients were divided according to whether the victim segment was evaluated by preoperative endoscopy + intraoperative inspection (PI group) or by intraoperative inspection (I group). In the PI group, suture repair of the victim segment was performed only when there was no evidence of disease on both preoperative endoscopy and intraoperative inspection. In the I group, whether suture repair was performed mainly depended on intraoperative findings (no obvious macroscopic disease such as stenosis, non-indurated tissue around the fistula, no ulcers on the mesenteric side, etc.).

\section{Definitions}

Postoperative complications were defined as any complication occurring within 30 days after surgery. Clinical recurrence was defined as a CDAI score $>150$ and CRP $>10 \mathrm{mg} /$ L. Endoscopic recurrence was defined as a Rutgeerts score of i2 or above. ${ }^{9}$ Surgical recurrence was defined as repeat resection for recurrent $\mathrm{CD}$.

\section{Surgical Technique}

All operations were performed by the same team using open laparotomy. The judgement of whether the victim segment was CD-free was based on preoperative examination and intraoperative inspection in the PI group, and repair was performed only when the victim segment was confirmed normal by both preoperative endoscopy and intraoperative inspection in this group, and by intraoperative inspection alone in the I group. After the fistula was divided by finger fracture and sharp dissection, the diseased segment was resected. Stapled side-to-side anastomosis or temporary fecal diversion was carried out depending on the patient's intraoperative presentation and general condition. The defect on the victim intestine was trimmed until soft, and then closed using a double-layer submucosal continuous transverse repair with 4-0 Vicryl absorbable suture (Ethicon, Johnson and Johnson Inc., Somerset County, NJ, USA). ${ }^{10}$ For temporary fecal diversion, delayed anastomosis was performed 3 to 6 months later. For maintenance therapy, azathioprine (AZA) was given within 2 weeks after surgery; if not tolerated, infliximab or Tripterygium wilfordii Hook F was considered. All patients were followed by internet, telephone, or outpatient visits. Endoscopic follow-up was initiated 2 to 6 months after surgery, and then performed every 6 to 18 months. For these patients, the victim segment was assessed if accessible on endoscopic examination.

\section{Statistics}

All analyses were carried out using GraphPad Prism version 6 (GraphPad Software, San Diego, CA, USA). $P$-values 
$<0.05$ were considered significant. Categorical data were expressed as frequencies and percentages, and continuous data were expressed as mean $\pm \mathrm{SD}$. The chi-square test was performed for group categorical data comparisons, and the $t$-test was used for group continuous data comparisons.

\section{RESULTS}

\section{Baseline Characteristics}

Of 65 patients ( 42 males and 23 females) who underwent intestinal repair during this period, 37 were in the PI group and 28 in the I group. The mean age at surgery was 37.75 years and the mean duration of disease was 80.46 months. The majority of patients belonged to A2 (46, 70.8\%), and L3 $(47,72.3 \%)$ phenotypes according to the Montreal classification. Twenty-one (32.3\%) had associated perianal disease, $10(15.4 \%)$ were smokers, and only 1 had a family history. Previous resection had been performed in $49.2 \%$. Nearly one-third received AZA preoperatively, while only $3.0 \%$ and $7.7 \%$ were treated with an anti-tumor necrosis factor (antiTNF) agent or steroid. Abdominal pain was the most common initial clinical manifestation, occurring in $63.1 \%$. About $75 \%$ (37/37 in PI group and 12/28 in I group, $P<0.0001)$ of fistulas could be diagnosed before surgery using radiography or endoscopy. Intestinal obstruction and failure of medical treatment were the top 2 indications for surgery, while fistulas were the sole indication for surgery in only 6 patients (9.2\%). Emergency surgery was performed in 5 patients $(0$ in PI group and 5 in I group, $P=0.012$ ). No other significant difference in baseline characteristics was found between PI and I groups (Table 1).

\section{Diagnosis of Fistulas and Evaluation of Victim Segments}

As all elective surgery patients ( 37 in PI group and 23 in I group) and 1 emergent surgery (perforation after endoscopy) patient in I group received preoperative colonoscopy, all sigmoid colon and rectum cases, except 4 requiring emergency surgery, were evaluated using endoscopy. Sixteen of 19 fistulas involving the duodenum could be diagnosed before surgery, and gastroduodenoscopy was performed in these patients. Due to difficult access, 3 fistulas could not be evaluated using endoscopy. Most fistulas between small intestinal segments could also be detected before surgery, but only 4 located on the terminal ileum could be reached and assessed using endoscopy (Table 2).

\section{Short- and Long-Term Outcomes}

The distribution of victim segments was different between PI and I groups. Due to inaccessible location on endoscopy, most small intestine fistulas were evaluated by intraoperative inspection. The size of the defect in the groups was similar.

Table 1. Baseline Characteristics

\begin{tabular}{|c|c|c|c|}
\hline Factor & $\begin{array}{l}\text { PI group } \\
(n=37)\end{array}$ & $\begin{array}{l}\text { I group } \\
(n=28)\end{array}$ & $P$-value \\
\hline Male sex & $24(64.9)$ & $18(64.3)$ & 0.831 \\
\hline Age at surgery (yr) & $37.95 \pm 10.50$ & $37.50 \pm 14.26$ & 0.885 \\
\hline Duration of disease (mo) & $84.27 \pm 46.67$ & $75.43 \pm 49.81$ & 0.466 \\
\hline \multicolumn{4}{|l|}{ Age at diagnosis } \\
\hline $\mathrm{A} 1$ & $1(2.7)$ & $4(14.3)$ & 0.156 \\
\hline $\mathrm{A} 2$ & $29(78.4)$ & $17(60.7)$ & 0.202 \\
\hline A3 & $7(18.9)$ & $7(25.0)$ & 0.775 \\
\hline \multicolumn{4}{|l|}{ Location } \\
\hline $\mathrm{L} 1$ & $4(10.8)$ & $5(17.9)$ & 0.483 \\
\hline L2 & $5(13.5)$ & $4(14.3)$ & 1.000 \\
\hline L3 & $28(75.7)$ & $19(67.9)$ & 0.676 \\
\hline Perianal lesions & $14(37.8)$ & $7(25.0)$ & 0.408 \\
\hline Smoking history & $4(10.8)$ & $6(21.4)$ & 0.306 \\
\hline Family history & $1(2.7)$ & 0 & 1.000 \\
\hline Previous resection & $20(54.1)$ & $12(42.9)$ & 0.520 \\
\hline Preoperative immunomodulators & $10(27.0)$ & $10(35.7)$ & 0.631 \\
\hline Preoperative anti-TNF & $1(2.7)$ & $1(3.6)$ & 1.000 \\
\hline Preoperative glucocorticosteroid & $5(13.5)$ & 0 & 0.065 \\
\hline Preoperative CRP (mg/L) & $20.71 \pm 38.74$ & $17.64 \pm 26.87$ & 0.721 \\
\hline Preoperative albumin (g/L) & $36.63 \pm 5.01$ & $33.78 \pm 7.33$ & 0.068 \\
\hline Preoperative BMI $\left(\mathrm{kg} / \mathrm{m}^{2}\right)$ & $17.99 \pm 2.37$ & $17.53 \pm 2.05$ & 0.421 \\
\hline Preoperative diagnosis of fistula & $37(100)$ & $12(42.9)$ & $<0.001$ \\
\hline Duodenum & $16(43.2)$ & $3(10.7)$ & 0.006 \\
\hline Small intestine & $4(10.8)$ & $9(32.1)$ & 0.058 \\
\hline Sigmoid colon/rectum & $17(45.9)$ & 0 & $<0.001$ \\
\hline \multicolumn{4}{|l|}{ Indication for surgery } \\
\hline Presence of mass or abscess & $6(16.2)$ & $5(17.9)$ & 1.000 \\
\hline Intestinal obstruction & $15(40.5)$ & $13(46.4)$ & 0.825 \\
\hline Failure of medical treatment & $11(29.7)$ & $9(32.1)$ & 0.835 \\
\hline Fistula & $5(13.5)$ & $1(3.6)$ & 0.225 \\
\hline Emergency surgery & 0 & $5(17.9)$ & 0.012 \\
\hline
\end{tabular}

Values are presented as number (\%) or mean \pm SD.

PI group, preoperative endoscopy + intraoperative inspection; I group, intraoperative inspection; TNF, tumor necrosis factor. 
Table 2. Diagnosis of Fistula

\begin{tabular}{lcc}
\hline Victim segment & $\begin{array}{c}\text { fistula diagnosed } \\
\text { before surgery/ } \\
\text { evaluated by endoscopy }\end{array}$ & $\begin{array}{c}\text { fistula found } \\
\text { intraoperatively }\end{array}$ \\
\hline Duodenum & $19 / 16$ & 6 \\
Small intestine & $13 / 4$ & 6 \\
Sigmoid colon/rectum & $17 / 17$ & 4 \\
Total & $49 / 37$ & 16 \\
\hline
\end{tabular}

There was no death after surgery. The rate of temporary diversion was high in this cohort (54.1\% in PI group and $46.4 \%$ in I group, $P=0.720$ ), but no permanent stoma was needed. Twelve cases ( 7 in PI group and 5 in I group, $P=1.000$ ) developed wound infections. One case in I group had anastomotic leakage, and 5 ( 3 in PI group and 2 in I group, $P=1.000$ ) had intra-abdominal abscess. Only 1 repaired defect (diameter, $4.0 \mathrm{~cm}$ ) in the duodenum in I group failed (leakage at the site of repair). This patient was treated conservatively for 6 months, and then received successful suture repair. No difference in stoma rates or incidence of postoperative complications was found between the groups (Table 3).

The mean duration of follow-up was 21.17 months in PI group versus 17.14 months in I group $(P=0.338)$. All patients received at least 1 endoscopic follow-up in this period. Overall, the rates of clinical, endoscopic, and surgical recurrence were $26.2 \%, 46.2 \%$, and $9.2 \%$ respectively. Among the 65 patients who underwent endoscopy, information about 3 repairs in PI group (2 in the duodenum and 1 in the small intestine) was not collected because of inaccessible location on endoscopy. Of these 3 patients, 2 were free from CD clinical and surgical recurrence and had no evidence of anastomotic recurrence. The other patient underwent reoperation due to anastomotic stenosis. No disease around the location of repair was observed in either group. No difference in longterm outcomes was found between the groups (Table 3).

\section{Risk Factors for Postoperative Complications}

To determine whether the location of the victim segment was a confounding factor for postoperative complications and identify other risk factors for complications, univariate analysis was carried out. No factor was found to be associated with postoperative complications: repair at duodenum $(P=0.579)$, repair at small intestine $(P=1.000)$, repair at sigmoid colon/rectum $(P=0.757)$, age at surgery $(P=0.544)$, duration of disease $(P=0.084)$, perianal lesions $(P=1.000)$, smoking history $(P=0.685)$, previous resection $(P=0.558)$,
Table 3. Operative Outcomes

\begin{tabular}{lccc}
\hline & $\begin{array}{c}\text { PI group } \\
(\mathbf{n = 3 7 )}\end{array}$ & $\begin{array}{c}\text { I group } \\
(\mathbf{n = 2 8 )}\end{array}$ & P-value \\
\hline Victim segment & $16(43.2)$ & $9(32.1)$ & 0.513 \\
\hline $\begin{array}{l}\text { Duodenum } \\
\text { Small intestine }\end{array}$ & $4(10.8)$ & $15(53.6)$ & $<0.001$ \\
\hline Sigmoid colon/rectum & $17(45.9)$ & $4(14.3)$ & 0.008 \\
\hline Diameter of detect (cm) & $1.12 \pm 0.80$ & $1.03 \pm 0.78$ & 0.664 \\
\hline Diversion & $20(54.1)$ & $13(46.4)$ & 0.720 \\
\hline Complications & & & \\
\hline Total & $10(27.0)$ & $9(32.1)$ & 0.862 \\
\hline Wound infection & $7(18.9)$ & $5(17.9)$ & 1.000 \\
\hline Failure of repair & 0 & $1(3.6)$ & 0.431 \\
\hline Anastomotic leak & 0 & $1(3.6)$ & 0.431 \\
\hline Abscess & $3(8.1)$ & $2(7.1)$ & 1.000 \\
\hline Duration of follow-up (mo) & $21.17 \pm 16.89$ & $17.14 \pm 16.02$ & 0.338 \\
\hline Clinical recurrence & $12(32.4)$ & $5(17.9)$ & 0.257 \\
\hline Reoperation for recurrence & $4(10.8)$ & $2(7.1)$ & 0.692 \\
\hline Disease at location of repair & 0 & 0 & $\mathrm{NA}$ \\
\hline V & 0 & \\
\hline
\end{tabular}

Values are presented as number (\%) or mean \pm SD.

PI group, preoperative endoscopy + intraoperative inspection; I group, intraoperative inspection; NA, not available.

preoperative immunomodulators $(P=0.762)$, preoperative anti-TNF agent $(P=1.000)$, preoperative glucocorticosteroid $(P=1.000)$, preoperative CRP $(P=0.600)$, preoperative albu$\min (P=0.071)$, preoperative diagnosis with fistula $(P=0.170)$, and emergency surgery $(P=0.076)$. Multivariate analysis was not performed because no risk factor was identified in univariate analysis.

\section{DISCUSSION}

Enteroenteric fistula is a common and intractable complication of $\mathrm{CD}$, and surgery is still the mainstay of treatment. The complexity of this type of fistula results from more extensive intestinal inflammation, associated abdominal mass, conjunction with the abdominal wall, or enterocutaneous fistula. As surgery is not curative for CD, $24 \%$ of patients need reoperation within 5 years. ${ }^{11}$ Surgical treatment with en bloc resection of fistulas may lead to the loss of a large quantity of normal intestine and will increase the risk of intestinal failure. As an internal fistula in CD is often a communication between diseased intestine and a victim intestinal segment, a more conservative procedure, with resection of only the diseased intestine and preservation of the victim segment, 
has been recommended for decades. ${ }^{12,13}$ Studies have confirmed this procedure is safe in CD ileosigmoid fistulas.

The main difficulty in the conservative procedure remains reliable diagnosis of the victim segment, especially in those fistulas found incidentally at the time of operation. If the victim segment affected by CD is left in place, an immediate (postoperative fistula) or late (recurrent disease) complication can develop. Endoscopy is the most useful examination for CD detection, and should be conducted if possible. CT can provide useful information for fistulas that are hard to reach on endoscopic examination and those found intraoperatively, but is not definitive. In the present study, we reviewed our experience of intestinal repair in fistulizing CD. Sixty-five repairs (25 in duodenum, 19 in small intestine, and 21 in sigmoid colon/rectum) were performed during a 5-year period. Results indicated that intraoperative inspection (macroscopic examination and tactile assessment) alone was a reliable guide when choosing between en bloc resection and a conservative procedure, and did not increase the risk of postoperative leak at the site of repair or postoperative disease recurrence.

Preoperative diagnosis of a fistula is important, and can help in the choice of appropriate examinations for the victim segment. Clinical symptoms and diagnostic methods such as endoscopy, CT, and barium enema are useful for the detection of internal fistulas. ${ }^{14,15}$ More than $70 \%$ of fistulas can be detected before surgery. Other than direct evidence for internal fistulas on diagnostic examination, studies have reported that a "star sign" on MRI or CT, and segmental sigmoid polyposis can also be used as diagnostic markers of enteroenteric fistulas. ${ }^{15,16}$

The value of preoperative endoscopic assessment of victim intestinal segments has been investigated in one study. Saint-Marc et al. ${ }^{8}$ compared 15 CD ileosigmoid fistula cases that underwent preoperative colonoscopy with 15 that did not, and found macroscopic intraoperative examination alone led to a $33 \%$ rate of incorrect evaluation of colonic involvement by $\mathrm{CD}$. There was greater morbidity without preoperative colonoscopy, but the difference was not significant. This result suggested that microscopic disease may not affect outcomes in the conservative procedure. In the present study, 28 fistulas were found intraoperatively or could not be reached on endoscopic examination. The assessment of the presence of $\mathrm{CD}$ in victim segments mainly depended on macroscopic observation and tactile assessment, and both short- and long-term outcomes of these repairs were also satisfactory. Preoperative endoscopic assessment should be done if possible, but a decision based on intraoperative inspection alone is acceptable for fistulas found incidentally during surgery and inaccessible with endoscopy.

Although the majority of patients received preoperative optimization, the rate of temporary fecal diversion in this study was $50.8 \%$, which was relatively high but consistent with previous reports $(7.7 \%-51.0 \%){ }^{7,17}$ Possible explanations are as follows. First, patients treated in our highly specialized inflammatory bowel disease center may be more complex. This was reflected in the presence of multiple fistulas, more intra-abdominal abscesses, and poor general conditions in this study cohort. Second, in order to reduce the risk of repair failure, surgeons intuitively preferred to perform a protective stoma. The incidence of postoperative complications was $29.2 \%$, and the incidence of intra-abdominal septic complications was $10.7 \%$. These results are superior to those reported in a meta-analysis $(20.2 \%)$ of open surgery in $\mathrm{CD},{ }^{18}$ but slightly higher than in a study that used similar preoperative management. ${ }^{17}$ Our results for clinical, endoscopic, and surgical recurrence are also comparable to those reported in a previous study. ${ }^{19}$ Postoperative AZA maintenance therapy, smoking cessation, and scheduled follow-up may contribute to the relatively low rate of recurrence.

This study has several limitations. First, because of the retrospective nature and small sample size, the results may be overestimated. Furthermore, this is a single-center study, and the surgical experience and practices may affect the results.

In conclusions, Enteroenteric fistula is a common complication of $\mathrm{CD}$. When surgery is required, a conservative procedure (resection of diseased segment and suture of the victim segment) is recommended, and the preoperative evaluation of the victim segment is important for the choice of surgical procedure. For fistulas found intraoperatively and inaccessible on endoscopic examination (preoperative endoscopic evaluation of the victim segment was not possible), intraoperative inspection (macroscopic examination and tactile assessment) alone was a reliable guide when choosing between en bloc resection and a conservative procedure, and did not increase the risk of postoperative leak at the site of repair or postoperative disease recurrence.

\section{FINANCIAL SUPPORT}

The authors received no financial support for the research, authorship, and/or publication of this article. 


\section{CONFLICT OF INTEREST}

No potential conflict of interest relevant to this article was reported.

\section{AUTHOR CONTRIBUTION}

W.Z. conceived and designed the study. Z.G. and X.C. collected and interpreted data and wrote the manuscript. R.L. collected data. J.G., Y.L., and L.C. analyzed the dataset.

\section{REFERENCES}

1. Scharl M, Rogler G, Biedermann L. Fistulizing Crohn's disease. Clin Transl Gastroenterol 2017;8:e106. doi: 10.1038/ctg.2017.33.

2. Yoon YS, Yu CS, Yang SK, Yoon SN, Lim SB, Kim JC. Intra-abdominal fistulas in surgically treated Crohn's disease patients. World J Surg 2010;34:1924-1929.

3. Bemelman WA, Warusavitarne J, Sampietro GM, et al. ECCOESCP consensus on surgery for Crohn's disease. J Crohns Colitis 2018;12:1-16.

4. Michelassi F, Stella M, Balestracci T, Giuliante F, Marogna P, Block GE. Incidence, diagnosis, and treatment of enteric and colorectal fistulae in patients with Crohn's disease. Ann Surg 1993;218:660-666.

5. Schraut WH, Chapman C, Abraham VS. Operative treatment of Crohn's ileocolitis complicated by ileosigmoid and ileovesical fistulae. Ann Surg 1988;207:48-51.

6. Young-Fadok TM, Wolff BG, Meagher A, Benn PL, Dozois RR. Surgical management of ileosigmoid fistulas in Crohn's disease. Dis Colon Rectum 1997;40:558-561.

7. Melton GB, Stocchi L, Wick EC, Appau KA, Fazio VW. Contemporary surgical management for ileosigmoid fistulas in Crohn's disease. J Gastrointest Surg 2009;13:839-845.
8. Saint-Marc O, Vaillant JC, Frileux P, Balladur P, Tiret E, Parc R. Surgical management of ileosigmoid fistulas in Crohn's disease: role of preoperative colonoscopy. Dis Colon Rectum 1995;38:1084-1087.

9. Rutgeerts P, Geboes K, Vantrappen G, Beyls J, Kerremans R, Hiele M. Predictability of the postoperative course of Crohn's disease. Gastroenterology 1990;99:956-963.

10. Gong J, Wei Y, Gu L, et al. Outcome of surgery for coloduodenal fistula in Crohn's disease. J Gastrointest Surg 2016;20:976-984.

11. Frolkis AD, Lipton DS, Fiest KM, et al. Cumulative incidence of second intestinal resection in Crohn's disease: a systematic review and meta-analysis of population-based studies. Am J Gastroenterol 2014;109:1739-1748.

12. Monson J. Surgical management is the preferred option for major internal fistulae. Inflamm Bowel Dis 2000;6:246-248.

13. Levy C, Tremaine WJ. Management of internal fistulas in Crohn's disease. Inflamm Bowel Dis 2002;8:106-111.

14. Brouquet A, Rangheard AS, Ifergan J, et al. The accuracy of preoperative imaging in measuring the length of the ileocolic segment affected by Crohn's disease: a prospective cohort study. Colorectal Dis 2017;19:437-445.

15. Braithwaite KA, Alazraki AL. Use of the star sign to diagnose internal fistulas in pediatric patients with penetrating Crohn disease by MR enterography. Pediatr Radiol 2014;44:926-931.

16. Korelitz BI, Taunk R, Kesar V. Segmental sigmoid polyposis as a colonoscopic indicator of an ileosigmoid fistula in Crohn's ileitis. J Crohns Colitis 2015;9:339-341.

17. Zerbib P, Koriche D, Truant S, et al. Pre-operative management is associated with low rate of post-operative morbidity in penetrating Crohn's disease. Aliment Pharmacol Ther 2010;32:459465.

18. Tan JJ, Tjandra JJ. Laparoscopic surgery for Crohn's disease: a meta-analysis. Dis Colon Rectum 2007;50:576-585.

19. Savarino E, Bodini G, Dulbecco P, et al. Adalimumab is more effective than azathioprine and mesalamine at preventing postoperative recurrence of Crohn's disease: a randomized controlled trial. Am J Gastroenterol 2013;108:1731-1742. 